\title{
Zinc Status in Virological Controlled Human Immunodeficiency Virus Type 1 Infected Patients
}

\author{
Carlos Tornero, Consuelo Lapuebla, Ana Ventura, Julian Diaz, Maricarmen Mafe
}

Internal Medicine Department and Laboratory, Hospital Gandia, Valencia, Spain.

E-mail: tornero_car@gva.es

Received August $1^{\text {st }}$, 2011; revised September 12 ${ }^{\text {th }}$, 2011; accepted September $24^{\text {th }}, 2011$.

\begin{abstract}
Zinc $(\mathrm{Zn})$ is a key micronutrient for correct immune function and its deficiency correction has been shown to be useful in HIV-infected but most of the studies included a significant proportion of patients without adequate virological control. It would be interesting to establish the prevalence, associated factors and clinical repercussions of Zn deficiency in patients with good virological to assessing the usefulness of $\mathrm{Zn}$ monitoring in the routine follow-up of well controlled $H I V$-infected patients, based on the colorimetric techniques commonly used in daily clinical practice. We included the first 100 patients that met the requirements of HAART and viremia levels under 200 copies for at least 6 consecutive months, with no active illicit drug use, active infections or weight loss of any cause during the previous 6 months. Serum Zn concentration was measured using a colorimetric assay (Sentinel Diagnostics ${ }^{\circledR}$ ) adapted to the Cobas 8000 analyzer (Roche Diagnostics). The Zn values showed a normal distribution with a mean concentration of $88.7 \mu \mathrm{g} / \mathrm{dl}$ (SD 23.3) and were found to be decreased in 13 patients and in 6 subjects were below $61 \mu \mathrm{g} / \mathrm{dl}$. Both the simple statistical analysis and the multivariate regression model only identified a significant effect for age and alcohol consumption. In sum an important number of HIV-infected with effective and prolonged HAART and no evidence of active infections or other associated factor show diminished serum Zn concentrations. The inclusion of at least occasional Zn determinations should be considered in the regular follow-up evaluations of HIV-infected patients.
\end{abstract}

Keywords: Zinc, Micronutrients, HAART

\section{Introduction}

Zinc $(\mathrm{Zn})$ is a key micronutrient for correct immune function. Zinc deficiency is not uncommon, and its correction has been shown to be useful in a number of clinical contexts [1]. HIV-infected patients often present Zn deficiency, and normalization of the levels of this element likewise offers benefit for such individuals [2]. However, most of the studies conducted in these subjects were published before highly active antiretoviral therapy (HAART) were available, and included a significant proportion of patients without adequate virological control, illicit drug users, and patients with active infections or malnutrition of different origins. It would be interesting to establish the prevalence, associated factors and clinical repercussions of $\mathrm{Zn}$ deficiency in patients with good virological control and without the aforementioned factors, with a view to assessing the usefulness of $\mathrm{Zn}$ monitoring in the routine follow-up of well controlled
HIV-infected patients, based on the colorimetric techniques commonly used in daily clinical practice.

\section{Material and Methods}

The systematic determination of $\mathrm{Zn}$ was introduced in all patients followed-up on in a center specialized in the management of HIV-infected individuals. We included the first 100 patients that met the requirements of HAART and viremia levels under 200 copies for at least 6 consecutive months, with no active illicit drug use, active infections or weight loss of any cause during the previous 6 months. Anthropometric data were collected, together with information relating to alcohol consumption, the presence of diarrhea, lipodystrophy, skin lesions, nadir and present CD4+ lymphocytes counts, transaminases levels and antecedents of decompensated liver cirrhosis. A total of 134 patients were seen until the planned 100 subjects were recruited (Table 1). 
Table 1. Baseline characteristics.

\begin{tabular}{lc}
\hline Age, mean years \pm SD & $44.5 \pm 10.05$ \\
Sex, \% males & 64 \\
CD4 cell count, mean years \pm SD & \\
Nadir & $218.1 \pm 172.1$ \\
Current & $640 \pm 357$ \\
Alcohol use \% participans & 13 \\
BMI, mean $\mathrm{kg} / \mathrm{m}^{2} \pm$ SD & $25.3 \pm 4.6$ \\
Receiving ART and with undetectable viral load $(<200$ copies $/ \mathrm{mL})$ & $100 \%$ \\
Serum zinc levels mean mcgr/dl , mean \pm SD & $88.7 \pm 23.3$ \\
\hline
\end{tabular}

Serum Zn concentration was measured using a coloriassay (Sentinel Diagnostics ${ }^{\circledR}$ ) adapted to the Cobas 8000 analyzer (Roche Diagnostics). The adult serum reference values were 66 - $150 \mu \mathrm{g} / \mathrm{dl}$. The SPSS version 13.0 statistical package was used to analyze the results, based on the Student t-test and Mann-Whitney U-test for categorical variables and using the Pearson correlation coefficient for quantitative variables. For the multivariate analysis we constructed a multivariate regression model with the $\mathrm{Zn}$ concentrations as response variable.

\section{Results}

The $\mathrm{Zn}$ values showed a normal distribution with a mean concentration of $88.7 \mu \mathrm{g} / \mathrm{dl}$ (SD 23.3). The levels were found to be decreased in 13 patients (13\%; 95\% CI 6 19), and in 6 subjects were below $61 \mu \mathrm{g} / \mathrm{dl}$. Both the simple statistical analysis and the multivariate regression model only identified a significant effect (R: 0.114, p < 0.01) for age (Cr: 0.15; 95\% CI $0.04-0.25$, p < 0.01) and alcohol consumption (Cr: 14.67; 95\%CI 1.49 - 27.85, p = 0.03 ), no differences being observed with respect to the nadir CD4+ lymphocytes count, present CD4+ lymphocytes count, body mass index, presence of diarrhea, lipodystrophy, transaminase elevation or diagnosis of liver cirrhosis. Zinc replacement therapy or dietary recommendations were provided in these subjects.

\section{Comments}

Zinc is a microelement obtained mainly from meat and legumes, and is absorbed in the jejunum and to a lesser extent in the large bowel, in relation to the plasma levels reached. Pancreatic enzyme alterations and diarrhea reduce $\mathrm{Zn}$ absorption, though the levels of this element are also found to be decreased in acute inflammatory processes, nutritional deficiencies, alcoholism, chronic liver disease and in intravenous drug abusers [1]. In view of the relationship between $\mathrm{Zn}$ and correct immune function, this element has been extensively studied in HIV-in- fected individuals, where $\mathrm{Zn}$ deficiency is commonprobably due to a coincidence of many of the above mentioned factors: lack of control of the infection [3], nutritional deficiency particularly in intravenous drug abusers [4], alcoholism and terminal liver diseases [5], diarrhea [3], etc. In turn, Zn deficiency in HIV-infected patients has been associated to increased viral replication and a poorer diagnosis $[3,6]$, and correction of such deficiency has been correlated to improvements in survival and immune recovery [3,6-8].

Studies in the HAART era have reported Zn deficiency in over $30 \%$ of all patients, with immunological benefits once the problem is corrected. However, in these studies the percentage of patients with virological control and the absence of other confounding factors is low [3,9].

The interest of our study is that it involves a group of patients with effective and prolonged HAART and no evidence of active infections or other associated factors, in which the $\mathrm{Zn}$ deficiency rate was found to be $13 \%$ with severe deficiency in 6\%. Among the mentioned factors associated to Zn deficiency, only at least moderate alcohol consumption was associated to diminished levels of the element-no correlation being observed with transaminase elevation, liver cirrhosis, lipodystrophy, diarrhea or CD4+ counts. Although we cannot rule out specific nutritional deficiencies, the latter were not clinically evident, since we did not include patients with recent weight loss, and the body mass index of the subjects with Zn deficiency was $25.3 \mathrm{~kg} / \mathrm{m}^{2}$ (SD 4.6). In contrast, we observed an inverse correlation to age not previously described in the literature, and which might be attributable to dietary differences. Possibly other factors, e.g., non-evident active infections such as hepatitis C, persistent immune activation phenomena inherent to HIV disease, or dietary or genetic factors conditioning absorption could explain some case of $\mathrm{Zn}$ deficiency [10]. The direct colorimetric method used in this study offers the advantages of being technically easier, automatically 
performed and less costly than the atomic absorption spectrophotometric techniques used in other studies, and as such is the method usually employed in clinical laboratories. As has been commented, the clinical relevance of Zn deficiency and the benefits derived from correcting the problem are difficult to establish in our case, because most of the patients had high CD4+ counts (640 cells $/ \mathrm{mm}^{3}$, SD 357) — with no differences versus patients without Zn deficiency.

In sum, an important number of HIV-infected patients in the HAART era show diminished serum Zn concentrations. In a large percentage of cases the underlying cause cannot be identified, since these subjects have good virological control, with no liver disease or malnutrition. Further studies are needed to establish the causes and corroborate the usefulness of $\mathrm{Zn}$ replacement therapythough given the high prevalence of Zn deficiency even in patients with good nutritional status and virological control, the inclusion of at least occasional Zn determinations should be considered in the regular follow-up evaluations of HIV-infected patients.

\section{REFERENCES}

[1] M. Hambridge, “Human Zinc Deficiency,” Journal of Nutrition, Vol. 130, Suppl. 5S, 2000, pp. 1344S-1349S.

[2] E. Mochegiani and M. Muzzioli, “Therapeutic Application of Zinc in Human Immunodeficiency Virus against Opportunistic Infections,” Journal of Nutrition, Vol. 130, 2000, Suppl. 5S, pp. 1424S-1431S.

[3] C. Y. Jones, A. M. Tang, J. E. Forrester, et al., "Micronutrient Levels and HIV Disease Status in HIV-Infected Patients on Highly Active Antiretroviral Therapy in the Nutrition for Health Living Cohort," Journal of Acquired Immune Deficiency Syndromes, Vol. 43, No. 4, 2006, pp.
475-482. doi:10.1097/01.qai.0000243096.27029.fe

[4] M. K. Bann, A. Campa, S. Lai, H. Lai and J. B. Page, "Zinc Status in Human Immunodeficiency Virus Type 1 Infection in Illicit Drug Use,” Clinical Infectious Diseases, Vol. 37, Suppl. 2, 2003, pp. S117-S123. doi:10.1086/375875

[5] J. P. Zarski, J. Arnaud, H. Labadie, et al., "Serum and Tissue Concentrations of Zinc after Oral Supplementation in Chronic Alcoholics with or without Cirrhosis," Gastroenterologie Clinique et Biologique, Vol. 11, No. 12, 1987, pp. 856-860.

[6] M. K. Baum, G. Shor-Posner, Y. Lu, et al., "Micronutrients and HIV-1 Disease Progression,” AIDS, Vol. 9, No. 9, 1995, pp. 1051-1056. doi:10.1097/00002030-199509000-00010

[7] E. Mocchegiani, M. Muzzioli, R. Gaetti, et al., "Contribution of Zinc to Reduce CD4+ Risk Factor for "Severe" Infection Relapse in Aging: Parallelism with HIV," International Journal of Immunopharmacology, Vol. 21, No. 4, 1999, pp. 271-281. doi:10.1016/S0192-0561(99)00009-0

[8] E. Mocchegiani, S. Veccia, F. Ancarani, et al., "Benefit of Oral Zinc Supplementation as an Adjunct to Zidovudine (AZT) Therapy against Opportunistic Infections in AIDS,” International Journal of Immunopharmacology, Vol. 17, No. 9, 1995, pp. 719-727. doi:10.1016/0192-0561(95)00060-F

[9] M. K. Bann, L. Shenghan, S. Sales, J. B. Page and A. Campa, "Randomized, Controlled Clinical Trial of Zinc Supplementation to Prevent Immunological Failure in HIV Infected Adults,” Clinical Infectious Diseases, Vol. 50, No. 12, 2010, pp. 1653-1660. doi:10.1086/652864

[10] B. Lönerdal, "Dietary Factors Influencing Zinc Absorption,” Journal of Nutrition, Vol. 130, 2000, pp. 1378S1383S. 